\title{
Agentes de Software como Herramienta para medir la Calidad de Servicio Prestado en un Sistema de Transporte Público Colectivo Urbano
}

\author{
Mauro Callejas-Cuervo, Helver. A. Valero-Bustos y Andrea. C. Alarcón-Aldana \\ Grupo de Investigación en Software, Escuela de Sistemas y Computación, Facultad de Ingeniería. \\ Universidad Pedagógica y Tecnológica de Colombia, Sede Central Tunja-Boyacá-Colombia Avenida \\ Central del Norte 39-115, Colombia. (e-mail: maurocallejas@gmail.com, hvalero@rocktmail.com, \\ acalarcon@gmail.com)
}

Recibido Dic. 16, 2013; Aceptado Mar. 4, 2014; Versión final recibida May. 2, 2014

\begin{abstract}
Resumen
Se presenta el diseño, modelado y simulación del comportamiento de un sistema de transporte público colectivo urbano en ciudades de tamaño mediano, a través de la cuantificación de variables complejas que miden la calidad del servicio prestado. El sistema se modeló usando la técnica "de abajo hacia arriba" (Bottom Up), el cual representa el comportamiento dinámico y estático basado en agentes de software. Además se describen los parámetros de entrada al software de simulación. Se presentan y discuten los resultados usando diferentes escenarios, los que permitieron el análisis de su comportamiento. El trabajo permitió deducir que los agentes de software pueden ser usados en el análisis microscópico de cualquier sistema complejo.
\end{abstract}

Palabras clave: agentes de software, simulación de sistemas complejos, calidad de servicio, transporte público

\section{Software Agents as a Tool for Measuring Quality of the Provided Service in a Common Urban Transportation System}

\begin{abstract}
This work presents the design, modeling and simulation of behavior of an urban transportation system of a medium size city by means of the quantification of complex variables that measure the quality of the service provided. The system was modelled by using the Bottom Up technique, which represents the dynamic or static behavior based on software agents. Also, the input parameters of the simulation software are described. The results are presented and analyzed using different scenarios allowing their behavioral analysis. This work allowed concluding that software agents can be used in the microscopic analysis of any complex systems.
\end{abstract}

Keywords: software agents, complex systems simulation, service quality, software modeling, public transport 


\section{INTRODUCCIÓN}

La programación de la operación de una ruta o de un conjunto de rutas en un sistema de Transporte Público Colectivo Urbano (TPCU), es un proceso en el cual influye una serie de factores relacionados con la oferta y la demanda que están variando continuamente en el tiempo y en el espacio, por esta razón, a su programación se deben realizar ajustes continuos durante un período de ejecución. Con lo anterior se quiere decir que el proceso de programar la operación no es exacto, sino que está sujeto a incertidumbres, pero el no hacerlo ocasiona serias desventajas en la calidad de la prestación del servicio, que se reflejan en efectos negativos, tanto económicos como sociales.

Muchos de los sistemas sociales complejos han usado diferentes técnicas de simulación para analizar su comportamiento y planear estrategias de mejora en los resultados perseguidos (Calderón y Lario, 2007), (Izquierdo et al., 2008), (Callejas et al., 2013), (Ceballos et al., 2013), entre dichas técnicas están los agentes de software y sistemas multi-agentes, que son programas a los cuales se les asocia un comportamiento y características propias, que permiten representar un sistema real complejo (Oliveira, 2012; Rohbogner et al., 2012; Wooldridge, 1997). De acuerdo con García-Valdecasas (2011), una de las ventajas de la simulación basada en agentes es que a partir de una estrucutra individual es posible identificar las acciones, creencias, deseos y oportunidades, para transformarlas en un esquema social que representa el nivel macro y micro de la realidad de un sistema, la cual es vista como el resultado emergente de las interacciones entre los agentes o partes que lo constituyen (Escobar et al., 2011).

La simulación de los sistemas de transporte ha permitido identificar algunos de los elementos y variables que se deben tener en cuenta para la programación y distribución de recursos en una localidad y/o escenario específico (Retamales et al., 2008), (Carbajal, 2013); aunque también es de mencionar que se ha presentado poca exploración a nivel computacional usando agentes de software en la medición de variables de comodidad y rapidez, principales componentes de un sistema complejo (Nigel y Troitzsch, 2005), que indican la calidad del servicio prestado en un sistema TPCU. El objetivo de este trabajo se fundamentó en el uso de agentes de software con comportamiento propio para diseñar, modelar y simular el comportamiento de la operación de un sistema de Transporte Público Colectivo Urbano en ciudades intermedias, usando la técnica Bottom-Up (Guasch y Piera, 2009).

Al igual que las metodologías orientadas a objetos ofrecen guías para la identificación de objetos y las interdependencias entre ellos, existen metodologías para el modelado y diseño de sistemas multi-agente, la mayoría fundamentadas en el Lenguaje de Modelado Unificado (UML), éstas son base para el desarrollo de software con agentes, entre otras se tienen: BDI, MAS-CommonKADS, GAIA, MASSIVE, INGENIAS; por otro lado, la simulación basada en agentes, presenta guías que muestran cómo se puede descomponer un problema de este tipo, y cómo afectará un diseño en el funcionamiento general del sistema; ese diseño puede realizarse desde dos perspectivas, Top-down o Bottom-up; esta última, se basa en una arquitectura de agentes reactivos, la cual modela de lo particular a lo general, ya que visualiza en primer lugar, el sistema en cada una de sus partes (agentes inanimados), luego se asocian reglas a cada uno de estos agentes (agentes animados), continuando con la reunión de sociedades de agentes (organización de agentes) y por último se genera cualquier tipo de análisis del comportamiento de dichos agentes o datos (Macal y North, 2006), técnica que fue tomada como base para el desarrollo del presente trabajo.

\section{CONSTRUCCIÓN DEL MODELO BASADO EN AGENTES}

La simulación basada en agentes plantea desarrollar las siguientes etapas: Contextualización y análisis del sistema a simular, Modelamiento del sistema, Crear la interfaz gráfica de usuario, Crear escenarios a experimentar, Simular los modelos con un software, Obtener resultados, Analizar resultados. A continuación se describe de manera breve cada una de las fases.

Contextualización y análisis del sistema a simular: En el caso de estudio, se parte bajo la premisa que el usuario tiene creada una necesidad de viaje, y éste debe realizar una serie de acciones para poder llegar al destino final. Empieza por desplazarse al paradero del cual parte (origen), al encontrarse en el paradero debe seleccionar una ruta de viaje, es decir planear cuál será su itinerario (ruta), selecciona un vehículo, si encuentra sillas disponibles se sienta o cada vez realiza esta misma acción hasta encontrar una disponible antes de la llegada al final del tramo, termina su recorrido en el paradero destino y por último se desplaza al lugar objetivo. Este es el proceso que debe seguir una persona que desee hacer uso del sistema TPCU.

Modelamiento del sistema con agentes: La implementación del modelo se desarrolló en la herramienta AnyLogic. En ésta se implementaron clases activas o agentes, entre los cuales se tiene: Despachador, Despacho, Paradero, PeriodoP, PeriodoT, Persona, Ruta, Sitio, Tramo, Vehículo, Viaje, con su estructura propia, comportamiento y paso de mensajes; además se maneja la obtención de datos externos (DSTPCU, 
DSTPCUaux, DSTPCUaux1, DSTPCUaux2, DSTPCUaux3), que permiten tener acceso a registros incluidos en una base de datos y que almacenan los diferentes escenarios con sus parámetros asociados y utilizados en la simulación.

La estructura de un agente en AnyLogic, se programa y gestiona en las siguientes secciones: "code", "animation", "Algorithmic function", "statechar". La sección code, describe el código fuente relacionado con la gestión del agente; en la sección statechart, se configura el comportamiento de cada uno de los agentes que están involucrados en el modelo, allí se representan los diferentes estados y transiciones que pueden adoptar los agentes interactivos; en la sección animation, se configuran acciones referentes a la interactividad del agente con otros dentro del sistema.

Como se puede ver en la figura 1, el área de trabajo del simulador está dividida en tres secciones, en la sección 1, están organizados los agentes y demás objetos programables del sistema; en la sección 2 , se diagraman o modelan las conexiones y comportamientos de los agentes y su contexto, y en la sección 3 , se incluye la programación asociada con los agentes y el comportamiento que se quiera representar.

Ecuaciones asociadas al modelo generado: La programación base del software de simulación usado es Java, razón por la cual se requiere declarar variables, tanto globales como locales y asociar a cada una de ellas las operaciones que representa el comportamiento de cada agente; debido a la extensión del modelo y código fuente del sistema. En la Tabla 1 se presenta algunas de las principales ecuaciones utilizadas para calcular los valores de comodidad y rapidez en un TPCU.

Crear la interfaz gráfica de usuario: La creación y configuración de la interfaz gráfica de usuario, se realiza también con AnyLogic, en la figura 2, se observa el entorno visual del simulador basado en agentes.

Tabla 1: Algunas de las principales ecuaciones utilizadas para calcular los valores de comodidad y rapidez en un Transporte Público Colectivo Urbano

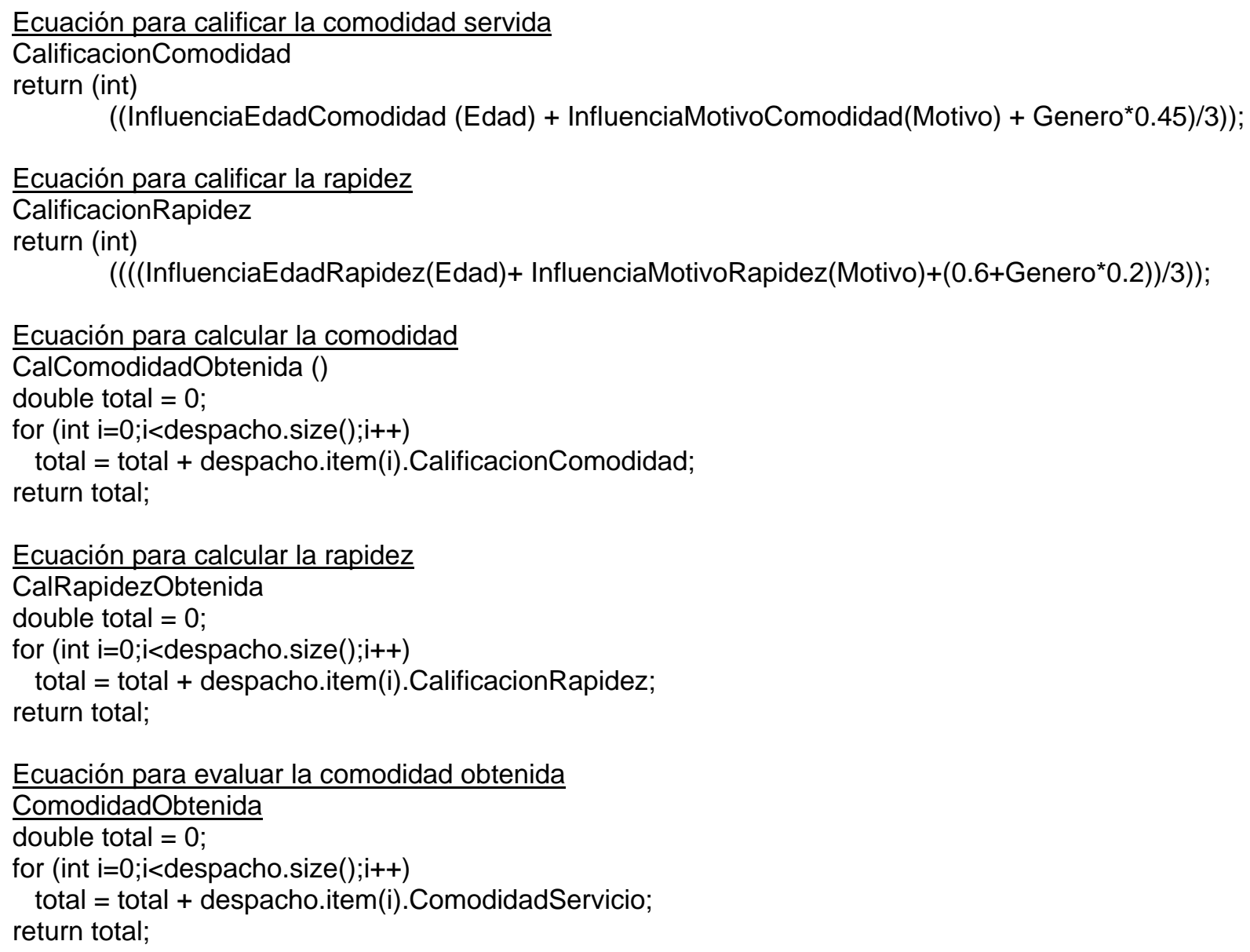




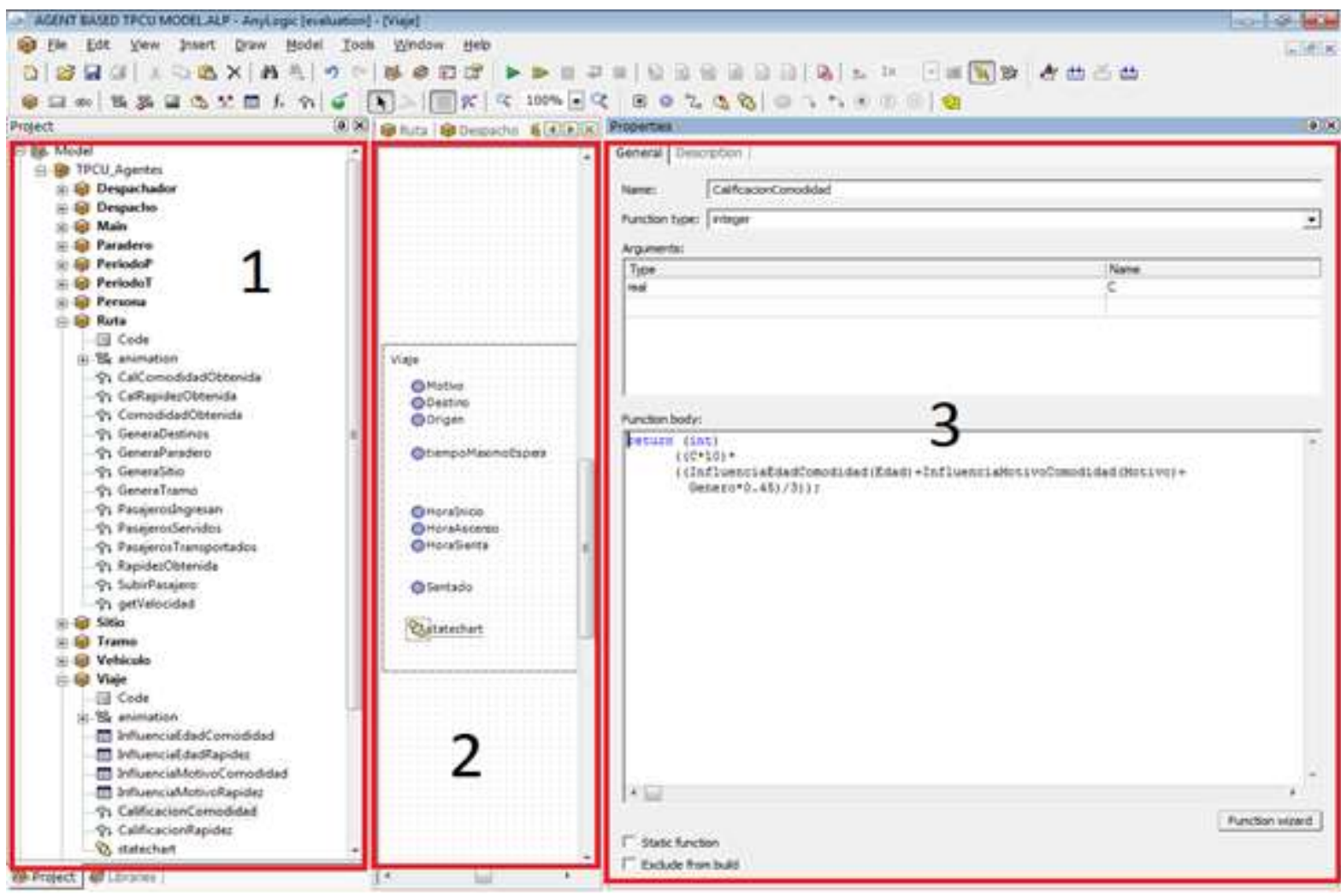

Fig. 1. División áreas de trabajo en AnyLogic.

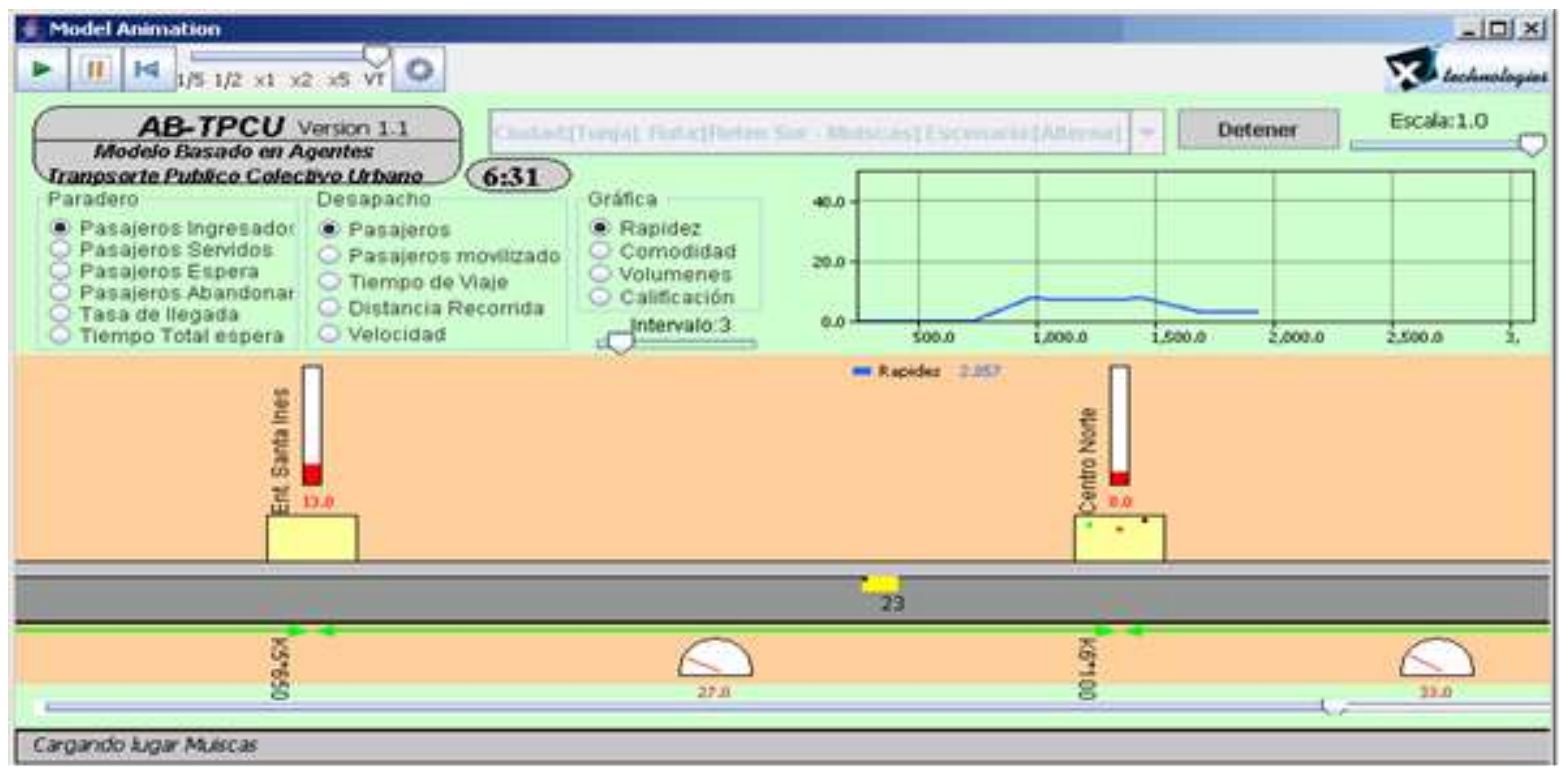

Fig. 2. Interfaz gráfica de usuario del simulador en agentes

Crear escenarios a experimentar: Para la creación de escenarios a experimentar se seleccionó la ciudad de Tunja, específicamente la ruta Reten Sur - Muiscas, que es una de las rutas más importantes que cubre la Ciudad de Sur a Norte. Se toman como base de análisis los datos obtenidos en el año 2003, ya que la infraestructura no ha cambiado considerablemente y son datos suministrados por fuentes de información confiables.

La ruta cuenta con catorce paraderos y tiene una longitud total de 8.370 metros, distribuidos así: 1) Reten Sur (Paradero Origen), 2)Los Hongos, 3) Comboy - Gaseosas, 4)Colegio Rosario, 5) K 9 Con Cll 21, 6) Nieves, 7) Hugolino, 8) Entrada Mesopotamia, 9) UPTC, 10) Santa Inés, 11) Centro Norte, 12) Toyota, 13) Asis, 14) Muiscas (Paradero destino). Escenario real: se tomó la programación de la ruta para un día hábil crítico, en la ciudad de Tunja, el cual es el día viernes, ya que en este día se incrementan las actividades económicas y sociales de la ciudad. La información de los parámetros base de simulación, se observa en la Tabla 2. 
Tabla 2. Escenario de simulación datos reales.

\begin{tabular}{|l|l|}
\hline Parámetro & Valor Parámetro \\
\hline Ruta & Reten Sur - Muiscas \\
\hline Día & Viernes \\
\hline Hora & 6 a.m. 10 p.m. \\
\hline Número de Vehículos & 27 Buses \\
\hline Despachos en el día & 65 Buses/Día \\
\hline Capacidad vehicular máxima día & 2500 Viajes \\
\hline Intervalo de tiempo de envío de despachos & 15 minutos \\
\hline
\end{tabular}

Escenario alterno: se parte de los valores mostrados en la Tabla 1, pero se disminuye el tiempo entre despachos (envío de vehículos para prestar el servicio de TPCU) en los periodos picos, es decir en horas comprendidas entre 7:00 a.m. y 9:00 a.m. (despachos cada 5 minutos) y en el periodo valle de 6:00 a.m. a 7:00 a.m. y de 9:00 a.m. a 11 a.m. se mantienen los mismos tiempos (despachos cada 15 minutos). En éste escenario se considera el mismo parque automotor de la programación real.

Simular los modelos con un software: Se simulan los modelos utilizando AnyLogic, tomando como base el escenario real y el escenario alterno. Para el análisis del comportamiento de las variables asociadas con la simulación basada en agentes, se toma un rango de la hora pico correspondiente a las 7:24 a.m. y 8:24 a.m. Es de anotar que en el modelo, la rapidez, comodidad y calificación, son registradas en el momento en que cada uno de los viajes (pasajeros) culmina su recorrido. Las fases de obtener y analizar resultados, se describen en detalle en la sección siguiente.

\section{RESULTADOS}

A continuación se describe el comportamiento de las variables rapidez y comodidad, tomando como referencia los escenarios real o base y el alterno; además se presenta el análisis de la evaluación de la calidad.

Variable Rapidez: Analizando la figura 3, literales a) y b), se concluye que la diferencia promedio de la rapidez entre los escenarios real y alterna es de aproximadamente tres kilómetros por hora $(3 \mathrm{Km} / \mathrm{h})$, lo que en términos de tiempo implica para los usuarios un ahorro de 3 minutos aproximadamente en el viaje. El tiempo ahorrado está relacionado únicamente con el tiempo de espera de los usuarios en el paradero, ya que las velocidades de desplazamiento de los vehículos es relativamente constante; además se presenta una mejora en el comportamiento de la rapidez en el escenario alterno.

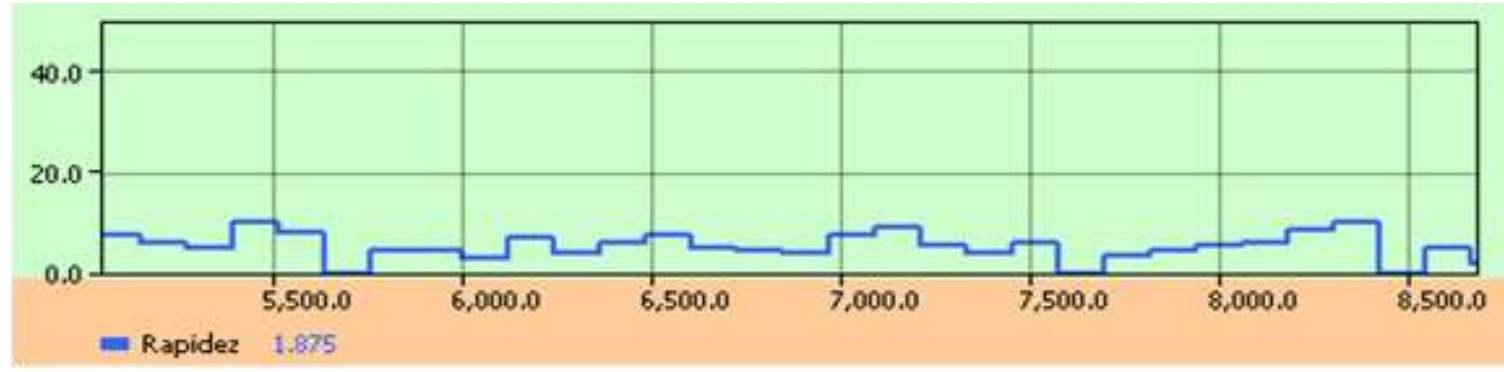

a) Variable Rapidez escenario real

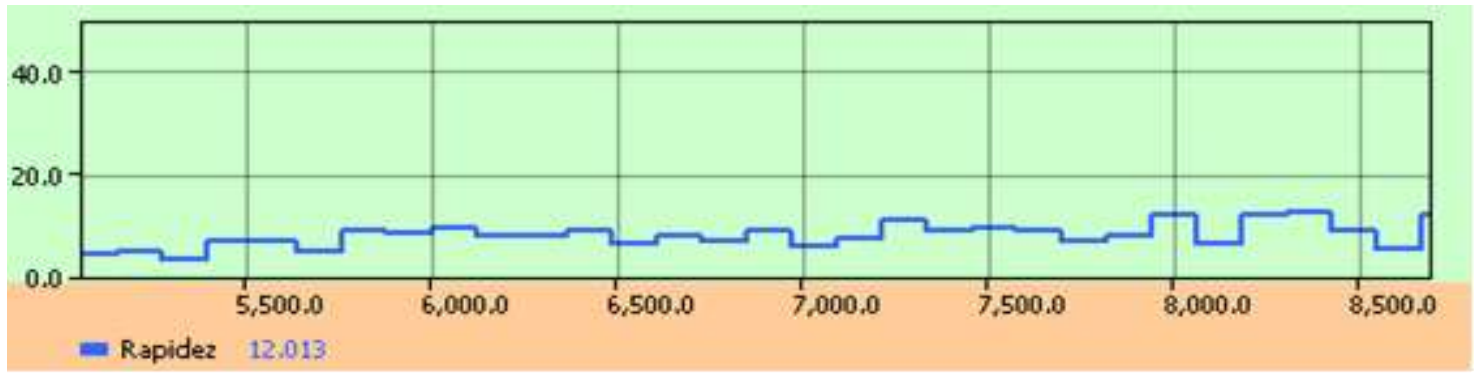

b) Variable Rapidez escenario alterno

Fig. 3. Comportamiento de la variable Rapidez. 
Variable Comodidad: La figura 4, literales a) y b), representan el comportamiento de la variable comodidad, donde se aprecia que existe un grado de comodidad mayor en el escenario alterno, ya que se aumenta la frecuencia de despachos y los usuarios tendrán una mayor posibilidad de viajar sentados.

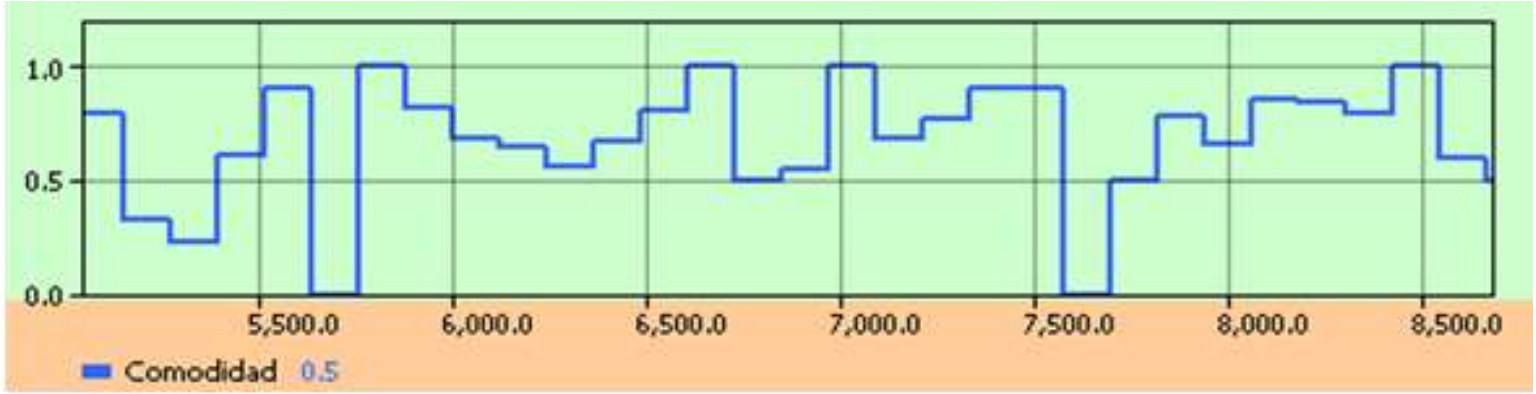

a) Variable Comodidad: escenario real

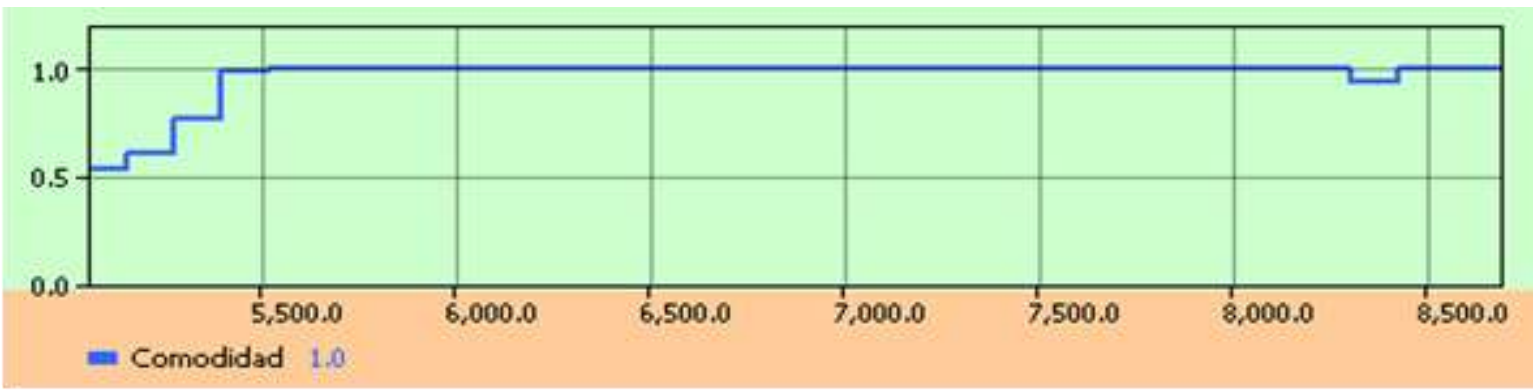

b) Variable Comodidad: escenario alterno

Fig. 4: Comportamiento de la variable Comodidad.

Calificación de la Calidad: La percepción del usuario de la calidad del servicio en cuanto a comodidad y rapidez, está dada en una escala de 0.0 a 10.0, donde 0.0 es una calidad deficiente y 10.0 es una excelente calidad. La figura 5, muestra este comportamiento, donde el escenario alterno presenta una trayectoria de la calificación más uniforme que el escenario real, reflejando que la calidad del servicio mejoraría con las condiciones configuradas en el escenario propuesto. Se nota además que la calificación de la comodidad en el escenario real es aproximadamente de 3.0 con una desviación alta: mientras que en el escenario alterno esta calificación es de 5.0 aproximadamente y con una desviación pequeña. Este mismo comportamiento se nota en la calificación de la rapidez, donde para el escenario real es de 1.5 y en el escenario alterno es de 2.5 .

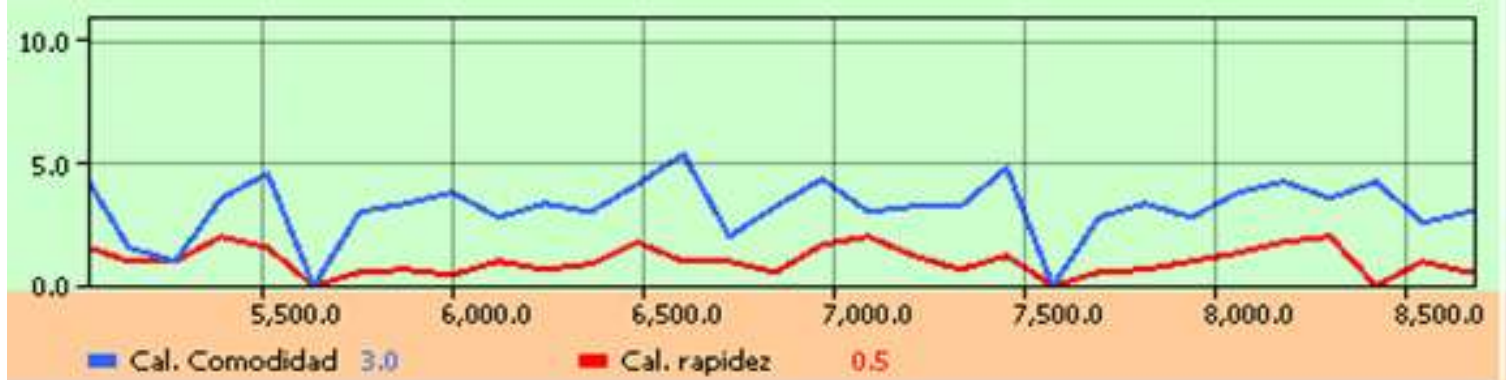

a) Calidad escenario real

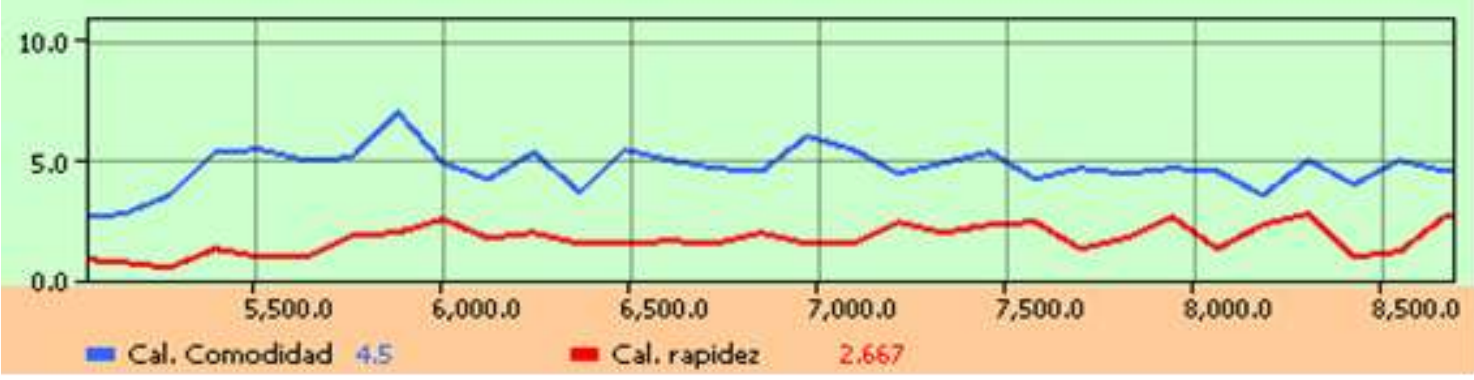

b) Calidad escenario alterno

Fig. 5: Calificación de la calidad del servicio prestado 
Visualizando el modelo se percibe la importancia del ajuste de la programación operativa del servicio TPCU, a cada uno de los diferentes periodos del día (periodos de máximo y periodos valle).

Los resultados promedio (condensando la información generada en un día por el sistema TPCU, en la situación real o base) por cada una de las variables de análisis fueron:

Nivel de servicio valorando la comodidad:

Nivel de servicio valorando la rapidez:

Calificación Definitiva del servicio:

3,15

Se dice que la prestación del servicio en el sistema TPCU es REGULAR.

Los resultados promedio en la simulación basada en agentes (condensando la información generada en un día en el sistema TPCU Alterno) por cada una de las variables de análisis respecto a los factores fueron:

Nivel de servicio valorando la comodidad: $\quad 3,90$

Nivel de servicio valorando la rapidez: $\quad 3,10$

Calificación Definitiva del servicio: $\quad 3,50$

Se puede concluir que la calidad de servicio prestada por el sistema TPCU es BUENA.

En la Tabla 3, se presentan los resultados obtenidos después de simular algunos otros escenarios variando los valores de tiempos de envío de los vehículos.

Tabla 3: Resultados de las diferentes ejecuciones del modelo

\begin{tabular}{|r|r|r|r|r|r|r|}
\hline Escenario & $\begin{array}{c}\text { Calificación } \\
\text { Comodidad }\end{array}$ & $\begin{array}{c}\text { Calificación } \\
\text { Rapidez }\end{array}$ & $\begin{array}{c}\text { Viajes Diarios } \\
\text { Generados }\end{array}$ & $\begin{array}{c}\text { Viajes } \\
\text { Diarios } \\
\text { Servidos }\end{array}$ & $\begin{array}{c}\text { Tiajes } \\
\text { Desertados }\end{array}$ & $\begin{array}{c}\text { Tiemp } \\
\text { Total De } \\
\text { Espera }\end{array}$ \\
\hline Real & 3,75339367 & 2,51066581 & 4024 & 3094 & 930 & 911 \\
\hline Real & 3,70138889 & 2,48484849 & 4080 & 3168 & 2747 \\
\hline Alterno & 3,76529517 & 2,47048301 & 4068 & 2795 & 1273 & 12904 \\
\hline Alterno & 3,76421886 & 2,46364291 & 4038 & 2778 & 1260 & 12740 \\
\hline Alterno 1 & 3,69901622 & 2,53443233 & 4021 & 3761 & 257 & 2544 \\
\hline Alterno 1 & 3,69370331 & 2,5317502 & 4013 & 3748 & 264 & 2611 \\
\hline Alterno 2 & 3,84995252 & 2,50617284 & 4051 & 2106 & 1945 & 19813 \\
\hline Alterno 2 & 3,84222846 & 2,46910112 & 4068 & 2136 & 1931 & 19658 \\
\hline
\end{tabular}

\section{CONCLUSIONES}

Es importante llevar a cabo un seguimiento, a través de la aplicación del modelado basado en agentes que aquí se desarrolló, a rutas que presentan problemas de circulación de viajes, con el fin de tomar alguna decisión sobre el mejoramiento del servicio a este sector de población y por ende redundar en beneficio de las empresas que prestan el servicio; ya que éste tipo de modelado permite llegar a un nivel de detalle preciso, como fue el caso de poder hacer el seguimiento de un vehículo a través de toda la ruta con el fin de controlar la velocidad y capacidad que ofrece dicho vehículo; por otro lado permitió analizar los diferentes paraderos y poder mostrar que una determinada programación afecta a éstos de diversas maneras en momentos variados del día.

La construcción de modelos de este tipo, junto con la implantación de técnicas para la recolección de información permanente del sistema, permite el monitoreo y evaluación del comportamiento del sistema constantemente, y así proporcionar las herramientas para tomar decisiones acertadas y a tiempo en lo relacionado con la programación de la operación del TPCU, además de permitir una primera aproximación a los sistemas de transporte inteligentes.

\section{REFERENCIAS}

Calderón, J. L. y Lario, F. C. Simulación de Cadenas de Suministro: Nuevas Aplicaciones y Áreas de Desarrollo. Rev. Información Tecnológica, (en línea), 18(1), 137- 146, (2007). 
Callejas-Cuervo, M.; Valero, H. A. y Alarcón-Aldana, A. C. Simulation based on system dynamics for evaluating the quality of transport service in a complex social system. Rev. Dyna, (en línea), 80(180), 33-40, (2013).

Carbajal, E.; Aragón, L. G.; Dávila, C. B. Optimización basada en simulación en un sistema de tránsito público masivo. Actas del 11th Latin American and Caribbean Conference for Engineering and Technology. Cancún, México, 14 - 16 de Agosto (2013).

Ceballos, Y.F.; Uribe, M. y Sánchez, G. Modelo de Dinámica de Sistemas para la Predicción del Comportamiento del Mercado Porcícola. Rev. Información Tecnológica, (en línea), 124(4), 117-124, (2013).

Charles M. Macal, C. M.; North, M. J. Tutorial on agent-based modeling and simulation part 2: how to model with agents. Proceedings of the 2006 Winter Simulation Conference. Monterey, California, USA, 3-6 de Diciembre (2006).

Escobar, A.; Moreno, J.; Múnera, S. Simulación basada en agentes de software para la evaluación de indicadores técnicos. Rev. Fac. Ing. Univ. Antioquia, (en línea), 58(1), 123-132, (2011).

García-Valdecasas, J. I. La simulación basada en agentes: una nueva forma de explorar los fenómenos sociales. Rev. Reis, (en línea), 136 (4), 91-110, (2011).

Guasch, A.; Piera, M. A. Modelado y simulación: aplicación a procesos logísticos de fabricación y servicios. 2ª edición, 40-42. Editorial Universidad Politécnica de Cataluña, España (2009).

Izquierdo, L. R.; Galán, J. M.; Santos, J. I. y Del Olmo, R. Modelado de sistemas complejos mediante simulación basada en agentes y mediante dinámica de sistemas. Rev. Empiria, 16(2), 85-112, (2008).

Nigel, G. y Troitzsch, K. G. Simulation for the Social Scientist. 1a edición, 67-69. Open University Press, New York, USA (2005).

Oliveira, E. Software Agents: Can we Trust Them?. Actas del IEEE 16th International Conference on Intelligent Engineering Systems. New University of Lisbon, Portugal, 13-15 de Junio (2012).

Retamales, H. E.; Moll, F. H.; Olguín, G. D.; González, G. A.; Rodríguez, J. L.; y Díaz, J. L. Modelo de simulación del sistema de transporte público de pasajeros de Gran Mendoza. Indicadores. Rev. de Ciencia y Tecnología, (en línea), 4(3), 64-74, (2008).

Rohbogner, G.; Fey, S.; Hahnel, U.; Benoit, P. y Wille-Haussmann, B. What the term Agent stands for in the Smart Grid Definition of Agents and Multi-Agent Systems from an Engineer's Perspective. Actas del Federated Conference on Computer Science and Information Systems. Institute of Electrical and Electronics Engineers. Wroclaw, Polonia, 9-12 de Septiembre (2012).

Wooldridge, M. Agent Based Software Engineering. IEE Proceedings Software Engineering. Institute of Electrical and Electronics Engineers. Reino Unido, Febrero (1997). 